\title{
Dynamique spatio-temporelle des feux de brousse dans le Parc National du Mbam et Djerem (Cameroun)
}

\author{
Christelle Dadem GUEGUIM ${ }^{1 *}$, N. Martin TCHAMBA ${ }^{1}$ et Corneille Roger FOTSO ${ }^{2}$ \\ ${ }^{1}$ Département de Foresterie, Faculté d'Agronomie et des Sciences Agricoles (FASA), \\ Université de Dschang Cameroun. \\ ${ }^{2}$ Wildlife Conservation Society (WCS), Programme pour la Biodiversité au Cameroun, Tél.: + 2372222026 \\ 45 ; B.P.: 3055 Messa-Yaoundé,E-mail :wcscameroon@wcs.org \\ *Auteur correspondant, E-mail: christelledadem@yahoo.fr; Tel: +237 677613673/655995187
}

\section{REMERCIEMENTS}

Nous tenons à remercier sincèrement la Wildlife Conservation Society et le projet d'Appui à l'Aménagement du Parc National du Mbam et Djerem qui nous ont fourni les moyens financiers et techniques pour cette étude.

\section{RESUME}

$\mathrm{Au}$ Cameroun, l'usage récurent et anarchique des feux de brousse, entraînant des dégâts sur l'écosystème, constitue une situation préoccupante pour les gestionnaires des aires protégées. Le Parc National du Mbam et Djerem (PNMD) est menacé par les feux de brousse et particulièrement par les feux tardifs incontrôlés. Cette étude visait à améliorer la compréhension du comportement des feux dans le PNMD. Spécifiquement, il s'agit d'analyser la distribution spatio-temporelle des feux actifs et des superficies brûlées dans le PNMD et de déterminer les principaux facteurs expliquent cette distribution. Pour y parvenir, les données satellitaires de moyenne résolution (MODIS : Moderate Resolution Imaing Spectroradiometer et Landsat), et les enquêtes de terrain ont permis de suivre l'évolution des feux de 2002 à 2015. La cartographie des feux actifs et des superficies brûlées est réalisée dans ArcGIS 10.1. Il s'en dégage une tendance à une périodicité des années de feux extrêmes de 1 à 2 ans depuis 2002. Mais, à partir de 2006, la tendance se maintient à un niveau bas. Le PNMD est plus vulnérables aux feux et concentrent près de $98 \%$ des feux actifs détectés sur toute sa superficie. Le taux annuel de superficie brûlée oscille entre 0.079 et $15 \%$ et entre 80 et $90 \%$ dans la zone tampon. $02,28 \pm 3,56 \%$ de sa superficie brûlent par an selon les données de télédétection. Cet outil de suivi et d'analyse spatio-temporelle des feux est valorisable pour la planification stratégique et opérationnelle au niveau local et national et pour une alerte précoce dans la gestion des feux de brousse.

(C) 2018 International Formulae Group. All rights reserved.

Mots clés: Aires protégées, conditions climatiques, MODIS Burned Area Product, surfaces brûlées, feux actifs, Savanes.

\section{Spatio-temporal dynamics of bush fires in the Mbam and Djerem National Park (Cameroon)}

\begin{abstract}
In Cameroon, the recurrent and anarchic use of bush fires, causing damage to the ecosystem, is a worrying situation for protected area managers. The Mbam and Djerem National Park (PNMD) is threatened by bush fires and particularly by uncontrolled late fires. This study aimed at improving the understanding of
\end{abstract}


fire behavior in the PNMD. Specifically, this involves analyzing the spatio-temporal distribution of active fires and burned areas in the PNMD and determining the main factors that explain this distribution. To achieve this, MODIS (Moderate Resolution Imaing Spectroradiometer and Landsat), and field surveys have been used to monitor fire trends from 2002 to 2015. The mapping of active fires and burned areas is performed in ArcGIS 10.1. There has been a trend towards a period of 1 to 2 years of extreme fire since 2002, but from 2006, the trend remains at a low level. The PNMD is more vulnerable to fire and concentrates nearly $98 \%$ of active fires detected over its entire area. The annual burned area rate varies between 0.079 and $15 \%$ and between 80 and $90 \%$ in the buffer zone. $02.28 \pm 3.56 \%$ of its area burns annually according to remote sensing data. This spatiotemporal fire monitoring and analysis tool is valuable for strategic and operational planning at local and national level and for early warning in the management of bush fires.

(C) 2018 International Formulae Group. All rights reserved.

Keywords: Protected area, climatic conditions, MODIS Burned Area Product, burned surfaces, active lights, Savannahs.

\section{INTRODUCTION}

Les feux de brousse sont des incendies qui parcourent chaque année les savanes africaines. Ce sont des phénomènes anciens dont la présence remonterait selon plusieurs auteurs (Valéa, 2010; Alvarado, 2012), au Néolithique. Ces feux sont présents dans la plupart des régions d'Afrique où ils jouent un rôle important de maintien en l'état ou de modification de la morphologie et de la composition spécifique du couvert. Mais, audelà de certaines normes de brûlages et de maîtrise des feux, l'aspect utilitaire des feux est supplanté par ses effets négatifs sur le couvert végétal et sur l'équilibre des écosystèmes (Archibald, 2008 ; Devineau et al., 2010; Kanvaly et al., 2011 ; N'dri, 2011 ; N'dri et al., 2011). Les feux incontrôlés exposent le sol à l'érosion et augmentent la charge atmosphérique en gaz à effet de serre (GES) et polluants (Pio et al., 2008 ; Ndedy et al., 2011 ; Vieira et al., 2015) et menacent ainsi la santé, la sécurité des biens et des infrastructures (Tarik et al., 2005).

Les feux, essentiellement d'origine anthropique (Hough, 1993 ; Poilecot et Loua, 2009 ; Opha, 2009), contribuent entre 13 et $40 \%$ à des émissions globales de gaz à effet de serre (GES) en équivalent carbone. Le phénomène tend à acquérir une importance particulière dans les aires protégées car le feu y est couramment utilisé pour le braconnage (Devineau et al., 2010; Kabore, 2010). Les gestionnaires des parcs, de même que les scientifiques sont ainsi convaincus qu'améliorer le contrôle de la pratique des feux est essentiel dans tout programme de conservation et de mise en valeur des aires protégées (Eva et al., 2003). Pour ce faire, il est indispensable de disposer d'informations quantitatives fiables sur l'étendue des surfaces brûlées, les types de couvert végétal touchés par le feu et enfin sur la saisonnalité des feux, au sein des aires protégées.

La répartition des feux de brousse est bien appréhendée par l'imagerie satellitaire (Giglio et al., 2003 ; Devineau et al., 2010 ; Valéa, 2010 ; Alvarado,2012). Elle permet d'observer les espaces où le feu se produit, et où il n'est pas présent. Elle montre aussi les périodes de passage des feux, ainsi que leur récurrence. Cette dynamique spatiale et temporelle est principalement déterminée par le milieu biophysique et les activités socioéconomiques (Valéa, 2010; Alvarado et al., 1014 ; Guiguindibaye et al., 2013).

Cependant, on opte actuellement pour la compréhension de la dynamique spatiotemporelle des feux pour espérer anticiper et minimiser le plus possible l'ampleur et l'intensité des incendies (Afelu, 2016). Les chercheurs se sont de plus en plus investis vers la connaissance à priori des zones à risques à travers l'utilisation des méthodes de télédétection et des Systèmes d'Information Géographique (SIG). Cette connaissance peut aider à une meilleure orientation des efforts de gestion en termes de prévention des feux et en termes d'évaluation des enjeux pour les populations (Collins et al., 2013). 


\section{MATERIEL ET METHODES}

\section{Zone d'étude}

La vulnérabilité spécifique des savanes des aires protégées (AP) a conduit cette étude à se focaliser sur le PNMD jugées prioritaires au niveau national. Le choix s'est porté sur cette aire protégée pour répondre : à une demande émanant de ONG Wildlife conservation society (WCS) qui finance le projet d'Appui à l'aménagement du PNMD / WCS ; à la demande des gestionnaires du parc qui considèrent le feu comme un des outils de gestion et de protection des terres (Grégoire et al, 2003); aux questions d'ordre scientifique que se posent les mêmes gestionnaires quant à l'impact des feux sur la nature et la structure du couvert végétal.

Le Parc National du Mbam et Djerem est situé entre $5^{\circ} 30^{\prime}$ et $6^{\circ} 13^{\prime}$ de latitude Nord, et $12^{\circ} 13^{\prime}$ et $13^{\circ} 10^{\prime}$ de longitude Est. Ce parc s'étend sur les régions du Centre, de l'Est et de l'Adamaoua, en plein cœur du Cameroun. Il se trouve à la limite méridionale du plateau de l'Adamaoua et en bordure nord de la forêt dense du bassin du Congo (Figure 1). On y trouve à la fois la forêt pluviale équatoriale (au Sud et à l'Est) et des zones de savanes de type soudano-guinéen avec une pluviométrie de $1800 \mathrm{~mm}$. Créé en janvier 2000, le parc couvre une superficie totale de 416512 ha, dont la moitié est constituée de forêt tropicale de plaine. L'autre moitié de savane est arborée et boisée. Entre les deux, on observe une large ceinture d'écotone, c'est-à-dire chevauchement des deux zones contigües. $\mathrm{Ce}$ chevauchement apporte au parc national du Mbam et Djerem une très grande diversité d'habitats et donc une grande biodiversité (MINFOF, 2012).

\section{Données satellitales}

Différents capteurs sont disponibles pour le suivi des feux actifs ou de surfaces brûlées et le niveau de résolution varie selon le capteur. Des images de faible résolution (la taille du pixel $\geq 1000 \mathrm{~m}$ ) sont proposées par les capteurs NOAA AVHRR et SPOT VEGETATION ; celles de moyenne résolution (la taille du pixel $\sim 250-500 \mathrm{~m}$ ) sont proposées par les capteurs MODIS et MERIS ; et celles de haute résolution (la taille du pixel $\leq 30 \mathrm{~m})$ sont proposées par le capteur LANDSAT, SPOT HRV / HRVIR, IKONOS, etc. L'information des images satellites des années avant 2000 sont disponibles seulement avec certains capteurs (exemple : NOAA AVHRR, SPOT VEGETATION ou LANSAT), ce qui rend difficile le suivi des feux pendant une période prolongée et supérieure aux vingt dernières années.

Notre choix s'est porté vers les images issues du capteur Modis qui dispose de caractéristiques techniques beaucoup plus intéressantes que ces concurrents. En effet, Modis produit des images qui peuvent atteindre la résolution spatiale de $250 \mathrm{~m}$, contre $1 \mathrm{~km}$ pour ses concurrents, en gardant une résolution temporelle journalière. La fréquence de passage (deux fois par jour) permet de capter un grand nombre de feux actifs et de détecter les surfaces brûlées.

Les feux dits "actifs", c'est -à -dire en activité au moment du passage du satellite, sont détectés par les systèmes d'observation TERRA et AQUA (http://terra.nasa.gov). Ces deux satellites orbitaux sont équipés, entre autres, de l'instrument MODIS qui est particulièrement bien adapté à la détection des feux actifs (Giglio, 2010).

Pour analyser la dynamique des feux de brousse, deux types de données ont été utilisées : les séries temporelles d'images MODIS des feux actifs de l'archive MCD14ML comme traité par le produit MOD14 Fire et Anomalies thermiques (Giglio, 2010) et mis à disposition par le Fire Information for Resource Management System (FIRMS). Ainsi que des séries temporelles d'images MODIS MCD45A1 correspondant aux produits des surfaces brûlées (Palumbo et al., 2011 ; Hantson et al., 2013) et téléchargées sur le portail (MODIS Burned Area Products -http://modisfire.umd.edu.) Chaque pixel de $250 \mathrm{~m}$ contient une information de qualité sur la présence ou non de surfaces brûlées et la date approximative du passage de feu pendant le mois analysé (Jacquin, 2010 ; Alvarado, 2012).

\section{Traitement des images}

Les données collectées ont été traitées dans le logiciel Excel version 2016 pour 
générer les histogrammes illustrés. Les cartes illustrant la dynamique spatiotemporelle des feux actifs et des superficies brûlées ont été réalisées dans le logiciel ArcGIS 10.1 dans le système WGS84 UTM Z33N. Les images MCD14ML et MCD45A1 sont exportées vers ArcGIS 10.1, en fichiers shapefiles puis superposés à la carte de la végétation du parc, les points de feux qui se trouvent à l'intérieur de la carte du parc sont ensuite découpés et classés de façon mensuelle afin de déterminer leur tendance saisonnière de distribution. Les zones de passage des feux et les zones de non feu ont été mises en exergue.

Dans la présente étude, 78 images mensuelles acquises entre le 2002 et 2015 ont été téléchargées du site web de l'USGS (http://terra.nasa.gov/) et traitées pour cartographier les surfaces brûlées (Tableau 1).

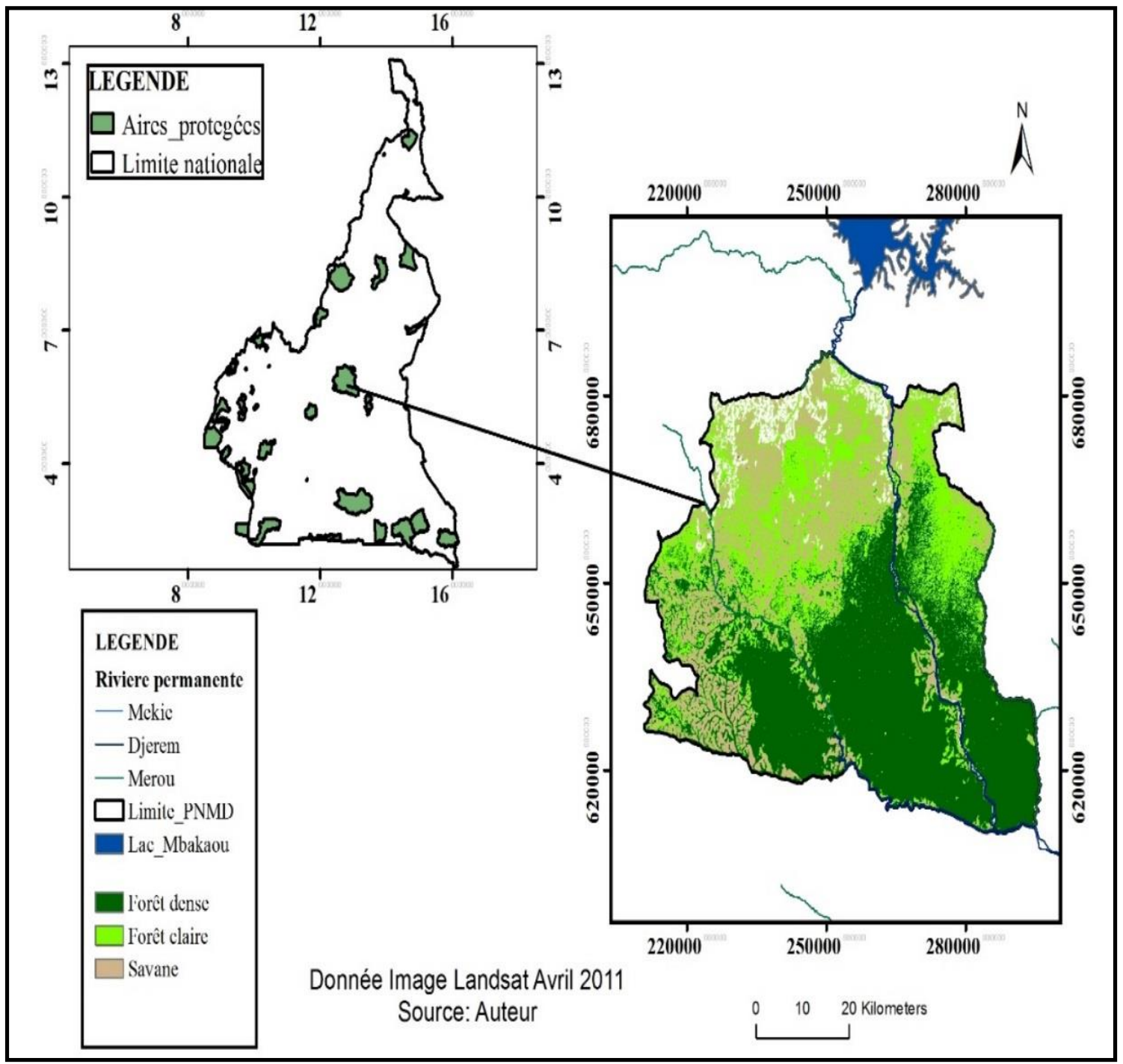

Figure 1: Localisation géographique de la zone d'étude. 
Tableau 1 : Différents sites de téléchargement et les paramètres d'acquisition des images.

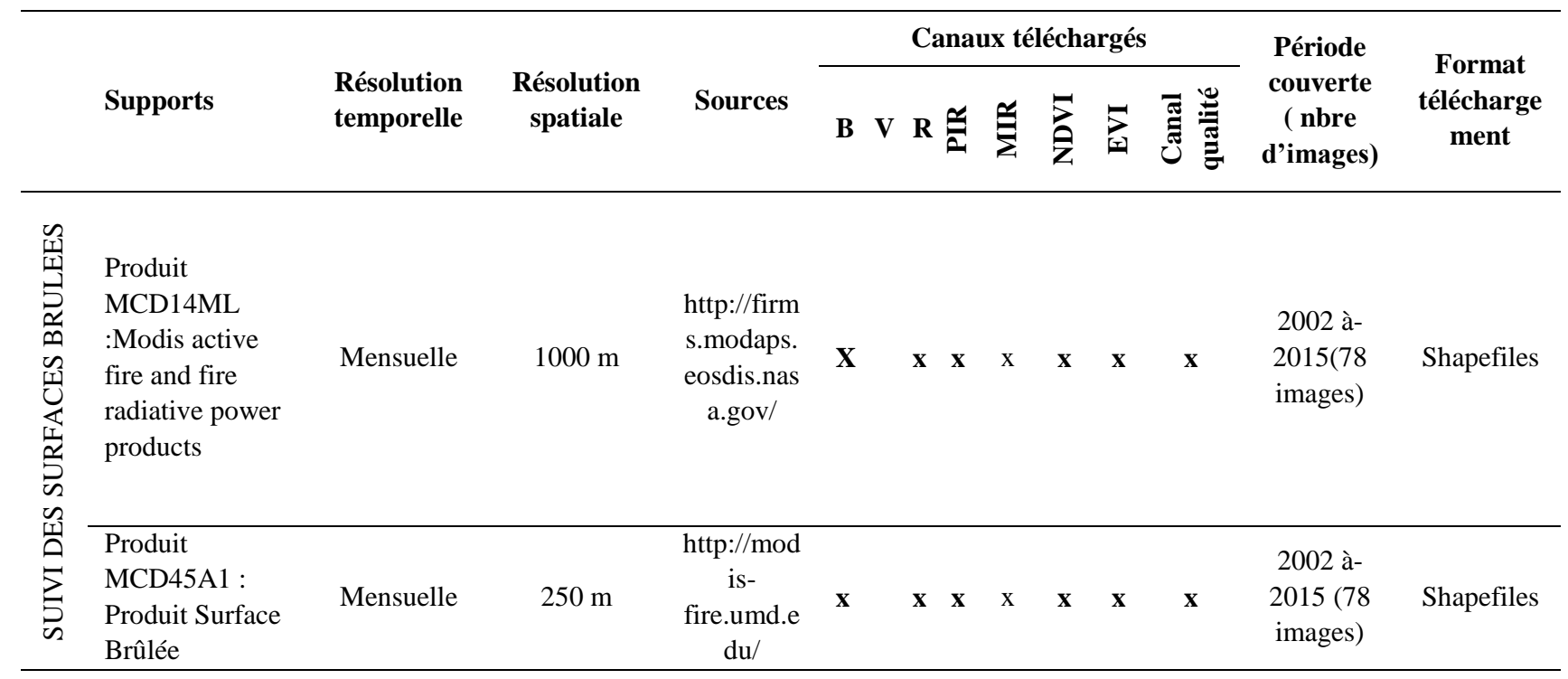

\section{RESULTATS}

Répartition spatiale et temporelle des feux actifs (2002-2015) dans le parc

Distribution spatiale des feux actifs dans le PNMD

L'analyse spatiale des feux a permis d'identifier l'espace de propagation des feux dans le PNMD (Figure 2) qui prend en compte les feux intervenus sur quatorze ans (novembre 2002 à avril 2015). Il ressort de cette Figure 2 que dans le PNMD la distribution des feux actifs n'est pas homogène. Cette disposition révèle que la propagation des feux dépend d'un certain nombre de facteurs qui favorisent ou non son passage qui est fortement influencée par les facteurs anthropiques. Cette dynamique des feux est alors interprétée comme le reflet de modes de gestion du parc. Ceci pourrait être dû à la non prise en compte de la mise a feux dans les activités de gestion du parc car le parc ne dispose pas encore d'un plan de gestion de feu et à la position géographique du PNMD qui le situe dans un climat qui varie du climat soudanien dans sa partie septentrionale, notamment à Mbakaou où on note la pluviométrie la plus élevée de la région dont $1800 \mathrm{~mm}$, au climat subéquatorial dans sa partie méridionale, constituée des forêts du
Centre et de l'Est un climat à saisons alternées. Dans ce domaine, le rythme climatique associe des mois de pluies à des mois secs dont la longueur varie en fonction de la position latitudinale. Cette saisonnalité agit sur le déroulement des feux : la saison pluvieuse correspond à la période où le feu est absent car l'état d'humidité de la végétation à cette période ne permet pas au feu de passer.

Le suivi annuel du nombre de feux actifs dans le PNMD de 2002 à 2015 révèle une moyenne annuelle de $577 \pm 43$ de feu actif. Le minimum a été observé durant la saison 2005-2006 (384 feux) et le maximum en 2003- 2004 (924 feux). La différence d'occurrence des feux actifs est significative d'une année à une autre $(p 0,027)$ et non entre les cycles de 2 et 4 ans (p 0,080 et 0,322). Ces résultats permettent de relever une tendance cyclique des années de feux extrêmes entre 1 et 2 ans (Figure 3).

Cette tendance cyclique entre des années de feux extrêmes peut s'expliquer par le fait qu'après un feu catastrophique qui a décimé la biomasse combustible une première année, l'écosystème mettrait 1 à 2 ans pour reconstituer la biomasse combustible suffisante pour une deuxième phase de feux intenses. 
Toutefois, il est noté que, depuis 2004, la tendance se maintient à un niveau bas. Ce maintien à un niveau bas des feux actifs détectés au niveau du PNMD depuis 2004 est dû beaucoup plus à la prise en charge du problème de gestion des feux par le service de conservation et du projet d'appui concernés et à l'efficacité de la lutte active car le parc est mieux surveillé. Il faut noter que c'est à partir de l'année 2000 et 2003, date de création du parc et d'arrivée du PAAPNMD/WCS, qu'on commençait à s'intéresser sérieusement à la protection de du parc ce qui expliquerait, probablement, la diminution des surfaces brûlées dans le parc et absence d'un plan de gestion des feux.

\section{Distribution temporelle des feux actifs (2002- 2015) dans le PNMD}

L'analyse de la distribution temporelle des feux a été faite sur l'ensemble des quatorze ans prenant en compte la variabilité interannuelle. Il en ressort qu'ils s'effectuent à un rythme régulier et cyclique (Figure 4). Ce cycle saisonnier des feux a permis de définir une «norme saisonnière » basée sur les mois propices au passage du feu qui coïncident avec la saison sèche où les pics de feux atteignent plus de 391 feux pour le mois de janvier. Cependant, le nombre total de feu reste variable d'une saison à l'autre.

La courbe des variations saisonnières des feux montre que la saison des feux s'étend sur cinq mois, de novembre à avril, comptabilisant environ $98 \%$ de tous les incendies (Figure 5). L'allure générale de la courbe des feux a une forme en cloche. Elle est caractéristique des courbes en zones tropicale à saisons alternées où les feux ne se propagent pas en saison pluvieuse du fait de l'humidité. Une analyse plus détaillée de la courbe annuelle des feux indique trois principales phases : le démarrage des feux, la saison des maximas des feux et la décroissance du nombre de feu.

Le démarrage des feux se situe au mois de novembre : cette phase correspond, pour la plupart, aux feux très précoces mis immédiatement après la saison des pluies. A cette période, la végétation n'étant pas assez sèche, ne permet pas au feu de se propager. La saison des maximas de feux correspond à la pleine saison sèche. Les occurrences de feux augmentent rapidement et atteignent leur maximum en janvier. L'accroissement des feux pendant cette période peut s'expliquer, d'une part, par le taux d'humidité de la végétation qui est au plus bas et, d'autre part, par la période d'intense activité dans le parc (élevage, chasse, aménagement).

La décroissance du nombre de feu commence en mars : à partir de cette période, on observe une décroissance régulière jusqu'en mai. Cette phase peut se justifier par le fait que les zones qui ont brûlé dans la deuxième phase ne sont plus susceptibles de brûler. Par ailleurs, le début de la saison des pluies en avril, dans certaines régions, limite les possibilités de mise à feu, mai marquent la fin de la saison des feux.

La distribution temporelle des feux fait apparaître des récurrences d'une année à l'autre. La répartition spatiale montre qu'ils ne sont pas présents partout à la même latitude. Ainsi les feux ne sont donc pas dus au hasard et leur fonctionnement est réglé par certaines variables qui permettent ou non son passage. Pour cela, il est nécessaire de comprendre pourquoi on observe des feux à certains endroits, ainsi qu'à des périodes précises.

\section{Distribution spatio-temporelle des superficies brûlées dans le PNMD}

Des superficies brûlées ont été enregistrées sur toutes les années de l'étude. Toutefois, la dynamique des feux varie d'une année à une autre. Proportionnellement à la superficie totale du parc, la superficie annuelle brûlée est en moyenne de $9516 \pm 5050$ ha. Depuis la saison 2005-2006, la superficie brûlée dans le parc se maintient à un niveau bas (moins de 4760 ha) jamais atteint depuis 2002. (Figure 6).

De l'intérieur de l'AP vers la périphérie et la zone tampon, la situation des feux n'est pas toujours la même dans le temps et dans l'espace. En effet, l'évolution du taux annuel de superficie brûlée montre qu'au 
niveau du PNMD, ce taux, qui oscille entre 0,079 et $15 \%$ de la superficie du parc, est significativement différent $(\mathrm{p}=0,02)$ de celui dans la zone tampon taux relativement élevé enregistré dans la zone tampon et compris entre 80 et $90 \%$ (Figure 7).

Le croisement SIG des couches de localisation des feux actifs, de la distribution des superficies brûlées et des différentes formations végétales au niveau du PNMD a montré que les feux concernent essentiellement les savanes (Figures 8). Les formations forestières au sens strict (écotone, forêt dense sèche, forêt claire, galerie forestière) en sont peu concernées. En discriminant les deux types de formation végétale (savanes et forêts), les superficies générées automatiquement montrent que, sur les quatorze saisons d'étude, les savanes représentent en moyenne $02,28 \pm 3,56 \%$ de la superficie brûlée dans PNMD.

\section{Déterminants de la propagation des feux dans le Parc National du Mbam et Djerem}

Le fonctionnement des feux est déterminé par trois facteurs : les conditions climatiques et le type de végétation; l'état du couvert végétal (état d'humidité, état de continuité) et les activités humaines.

\section{Conditions climatiques et le type de végétation}

Le climat impose ses périodicités et ses rythmes aux feux, à travers le comportement des pluies. La Figure 9 présente la courbe pluviométrique (en haut) et courbe de l'occurrence des feux (en bas) du PNMD. On observe sur cette figure que lorsque la saison sèche s'installe, les pluies se raréfient et les feux apparaissent. Ainsi la longueur des saisons influe la répartition des feux. Plus la saison pluvieuse est longue, moins on a des « mois de feux.

\section{Etat du couvert végétal (état d'humidité, état de continuité)}

La disposition latitudinale des pluies a une influence sur la disponibilité de la biomasse herbacée ainsi que sur la structure du tapis herbacé. Elle entrâne un raccourcissement du cycle végétatif au fur et à mesure de la progression latitudinale et finalement un éclaircissement du couvert végétal plus ou moins parallèle à la raréfaction des pluies. La Figure 10 présente les feux de brousse, conditions climatiques et état du couvert végétal entre 2002 et 2015 dans le PNMD. Il ressort de cette figure que la disposition latitudinale des pluies a une influence sur la disponibilité de la biomasse herbacée ainsi que sur la structure du tapis herbacé.

On observe une abondance d'herbacées pérennes au Nord du Parc qui favorisent le feu et au centre, des herbacées annuelles et peu abondantes.

L'état de continuité du couvert végétal conditionne la distribution des feux. Elle est en partie fonction de l'utilisation de la ressource par les sociétés. Dans les endroits très anthropisés, le couvert végétal est discontinu, le feu ne peut donc passer. Par contre, dans les zones à couvert végétale continue, les pratiques de sociétés n'ont pas entrainé une dégradation du couvert végétal, la continuité de la strate herbacée permet au feu de circuler.

\section{Populations dans la distribution des feux}

Le choix dans la mise à feu est conditionné par les variations de biomasse ; mais ce sont les populations qui déclenchent les feux avec des objectifs et un calendrier précis, en rapport le plus souvent avec leurs activités. Les populations agissent dans la propagation des feux en tant qu'élément déclencheur du feu et à travers les activités socio-économiques et culturelles qui autorisent ou non la mise à feu. La Figure 11 illustre les proportions des acteurs de mise à feux de brousses dans le PNMD. Il ressort de ce graphique qu'au PNMD, comme partout dans les milieux de savane, ce sont les populations à majorité pastorales, d'aménagements et braconniers, qui allument les feux pour plusieurs raisons : pratiques pastorales et renouvellement des repousses ou de chasse.

Le Tableau 3 présente les mois de mise à feu, les types de feu, les avantages pour les acteurs et inconvénients pour la faune. Il 
ressort de ce tableau que pendant les mois de novembre, décembre, janvier, février, mars et avril il existe deux grands types de feux dans le PNMD. Ce sont les feux précoces et les feux tardifs. Les feux tardifs sont pratiqués à plus de 98\%. L'élevage a une incidence sur la distribution des feux par la pratique de l'élevage à la périphérie du parc qui influence la présence de biomasse herbacée. C'est ce qui explique souvent, la présence de feu dans le parc, domaine de prédilection d'élevage où les feux sont utilisés comme un outil dans un contexte de disponibilité du couvert végétal. Par contre, l'absence de feu dans le nord s'explique par l'absence de l'activité pastorale qui, dans ces milieux, où l'on note une forte discontinuité végétation herbacée.

\section{Influence climatique sur le régime des feux}

Les analyses de corrélation croisée entre la fréquence des incendies, respectivement la surface brûlée annuelle, et l'indice de Thornthwaite ont révélé des résultats différents selon chaque année dans le PNMD (Tableau 2). Ce tableau montre que pendant les périodes 2002-2004 la fréquence des incendies était négativement et significativement corrélée avec l'indice de Thornthwaite durant la même année, cela n'était plus le cas de la période 2005-2015. A l'inverse, on observe pour la période 20052015 une corrélation positive entre la fréquence des incendies et l'indice avec un décalage de trois ans, c'est-à-dire que quand des conditions humides (donc un indice élevé) prévalaient une année, le nombre d'incendies augmentait significativement trois ans plus tard. Aucune corrélation significative de ce type n'est perceptible pour la période 2002 2004.

La Figure 12, établie pour la région du PNMD, montre bien qu'il y une relation claire entre la quantité de pluies tombées et les superficies brûlées, avec un coefficient de corrélation de $-0,56$. Plus les précipitations annuelles sont importantes, moins les superficies brûlées sont grandes. Toutefois, avec le réchauffement climatique prédit, les conséquences d'un déficit pluviométriques, risquerait d'être plus dramatique sur le couvert végétal du PNMD.

Annuellement, le PNMD perd, en moyenne, de 9410,05 à plus de 6257,68 hectares de superficie boisée (forêts, savanes, broussailles et steppes). Cette superficie brûlée présente une certaine variabilité annuelle en fonction de plusieurs facteurs que ce soit d'ordre météorologique (précipitation, température, vent) que biotique (type et structure du combustible).

Un bilan des incendies effectué dans le PNMD montre que le nombre de feux est en hausse depuis la création du parc bien que la superficie brûlée ait diminuée. Sur la Figure 12, on a observé qu'à partir de l'année 2005 , bien que le nombre de feu ait augmenté, les superficies brûlées sont moins importantes. Ceci est dû beaucoup plus à la prise en charge du problème de gestion des feux par le service de conservation et du projet d'appui concernés et à l'efficacité de la lutte active car le parc est mieux surveillé. Il faut noter que c'est à partir de l'année 2000 et 2003, date de création du parc et d'arrivé du PAAPNMD/WCS, qu'on commençait à s'intéresser sérieusement à la protection de du parc ce qui expliquerait, probablement, la diminution des surfaces brûlées dans le parc et absence d'un plan de gestion des feux.

La répartition de la fréquence des feux dans le temps $(2002$ - 2015) est à mettre en relation avec la variation du climat, qui se manifeste par une augmentation de la quantité de pluie et une diminution de la température au cours de ses 14 dernières années. Ceci se justifie par sa position géographique qui est située dans un climat qui varie du climat soudanien dans sa partie septentrionale, notamment à Mbakaou dans la région de l'Adamaoua où on note la pluviométrie la plus élevée de la région dont $1800 \mathrm{~mm}$, au climat subéquatorial dans sa partie méridionale, constituée des forêts du Centre et de l'Est un climat à saisons alternées. 


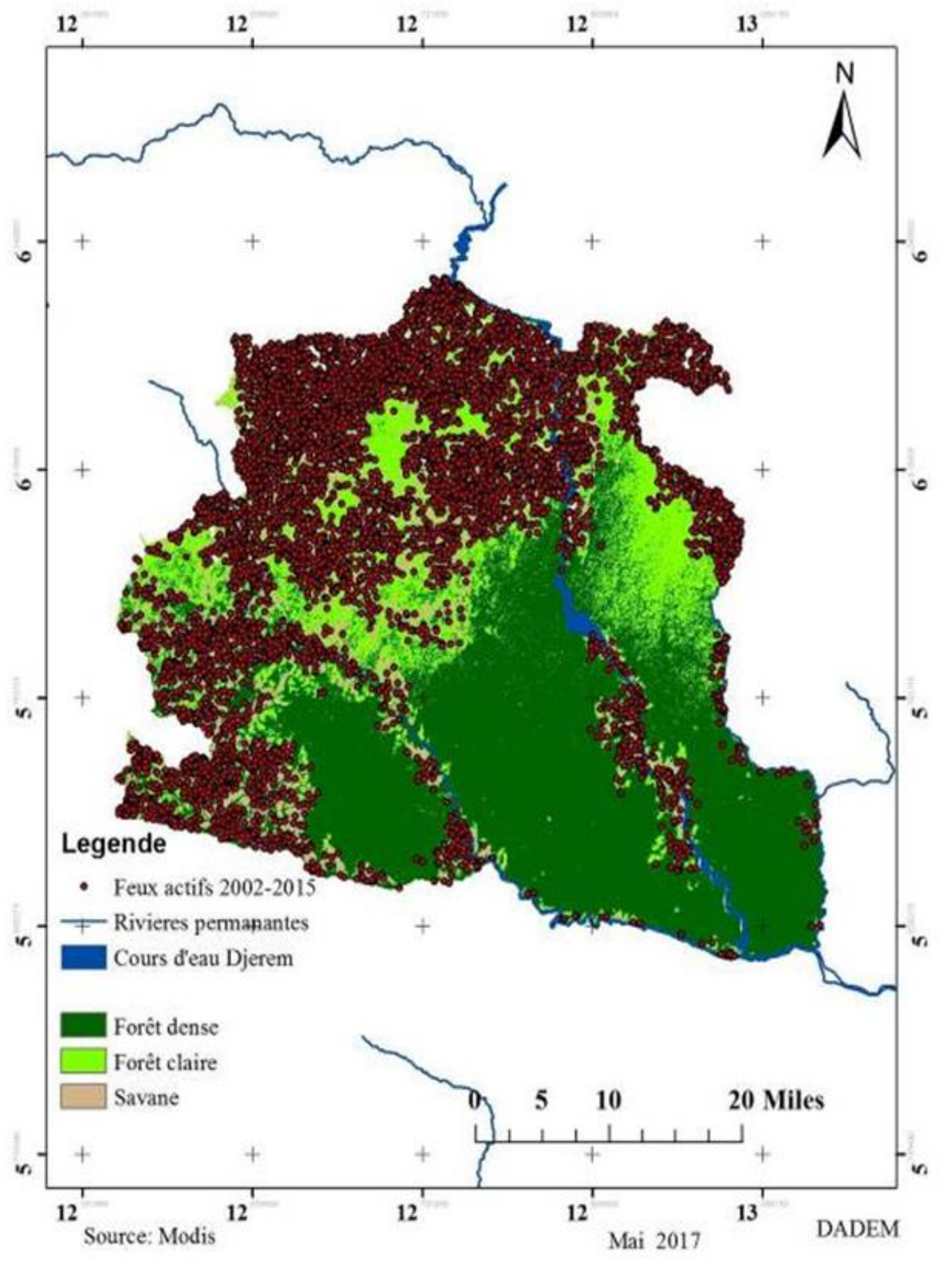

Figure 2: Fréquence des feux actifs et superficie brûlée dans le PNMD entre 2002 à 2015. 


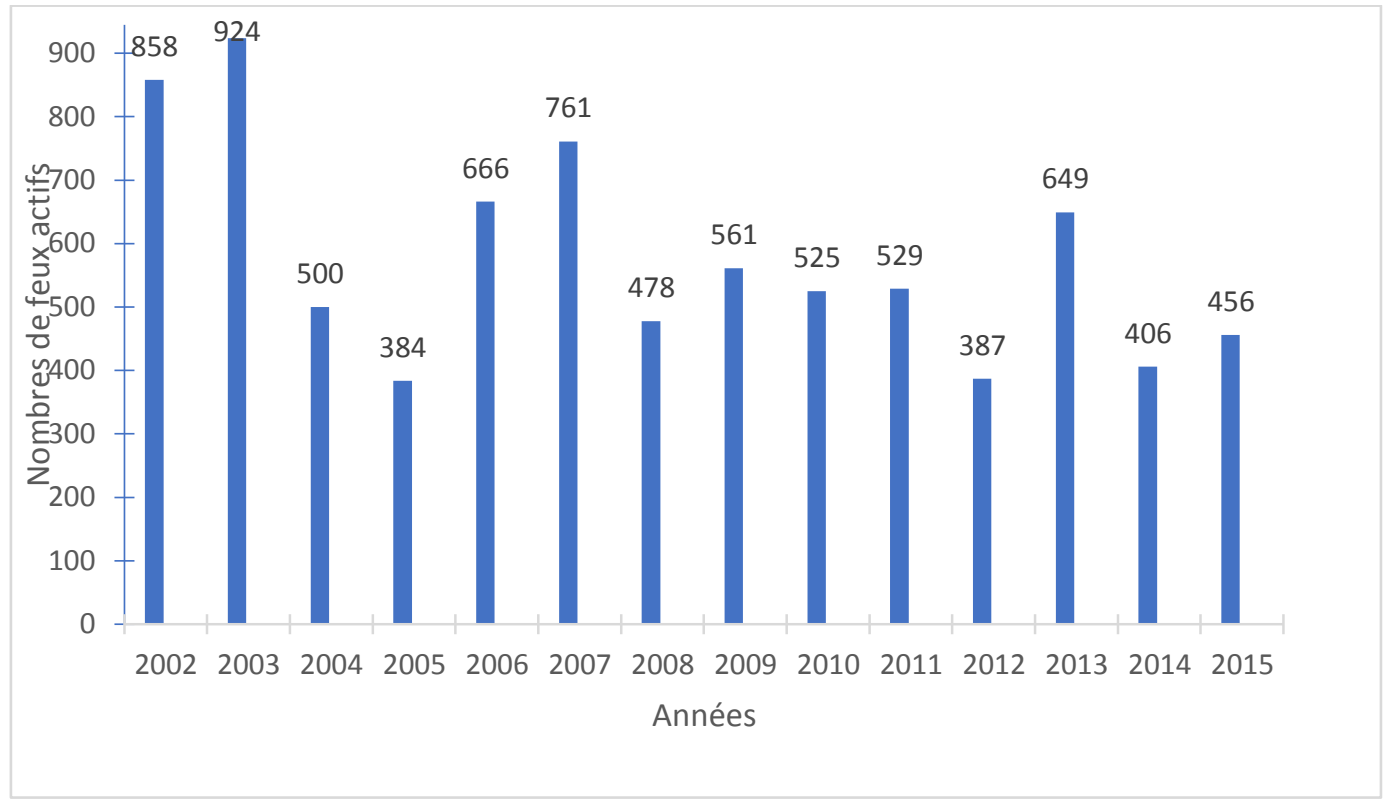

Figure 3: Evolution annuelle des feux actifs dans le PNMD.
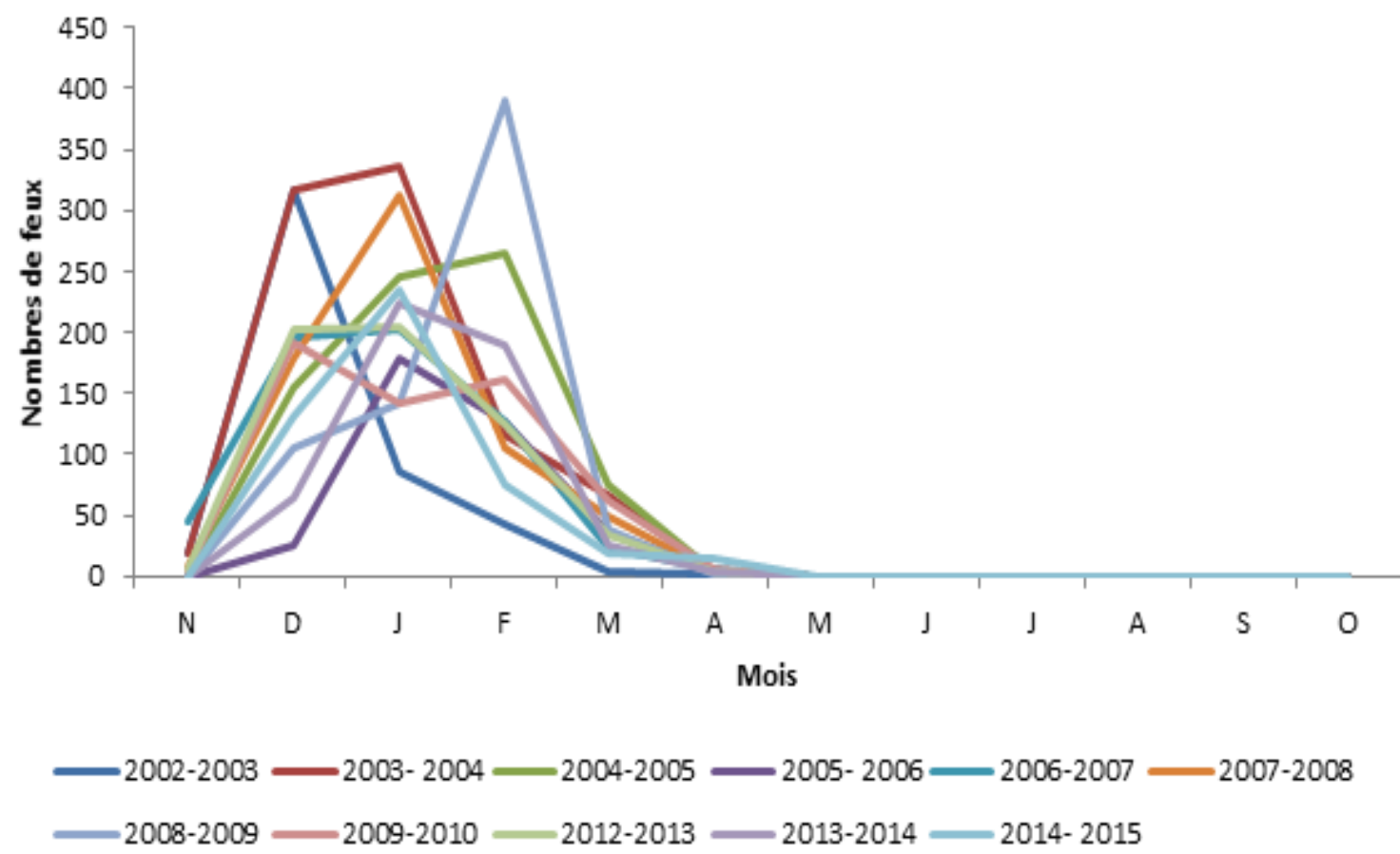

Figure 4: Répartition saisonnière de l'occurrence des feux pour les périodes 2002 à 2015 dans le PNMD. 


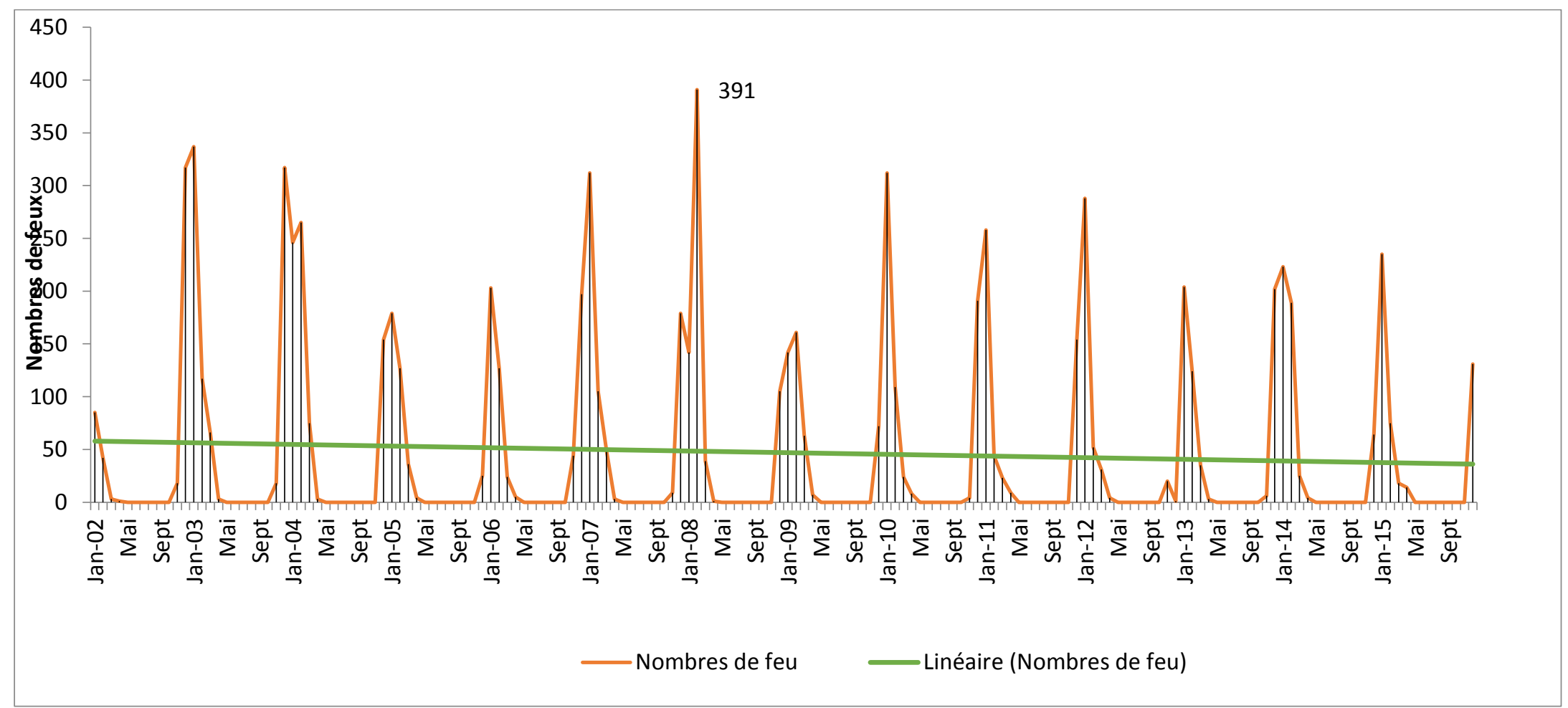

Figure 5: Variation interannuelle de l'occurrence des feux dans le PNMD. 


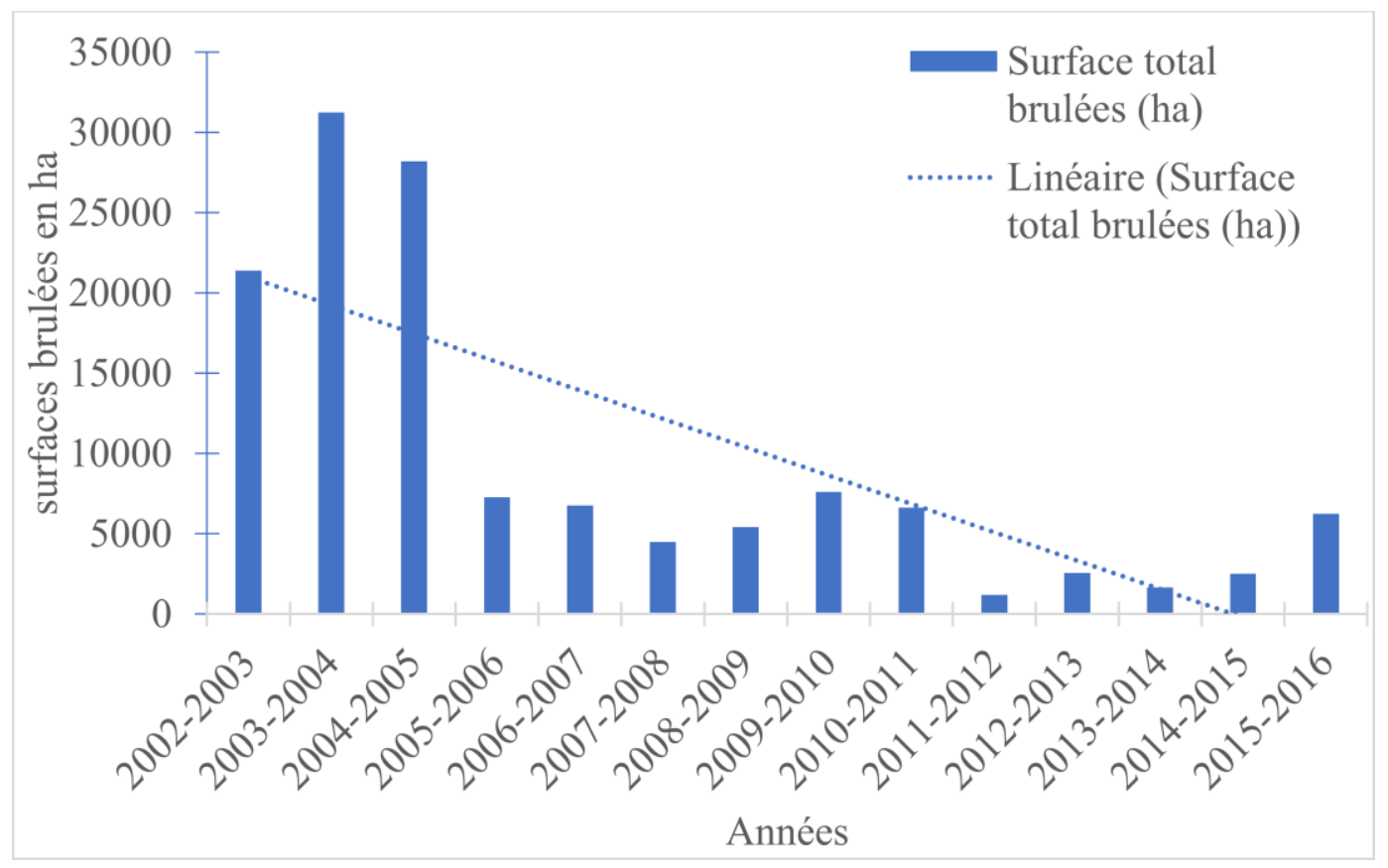

Figure 6: Evolution annuelle des superficies brûlées dans le PNMD.

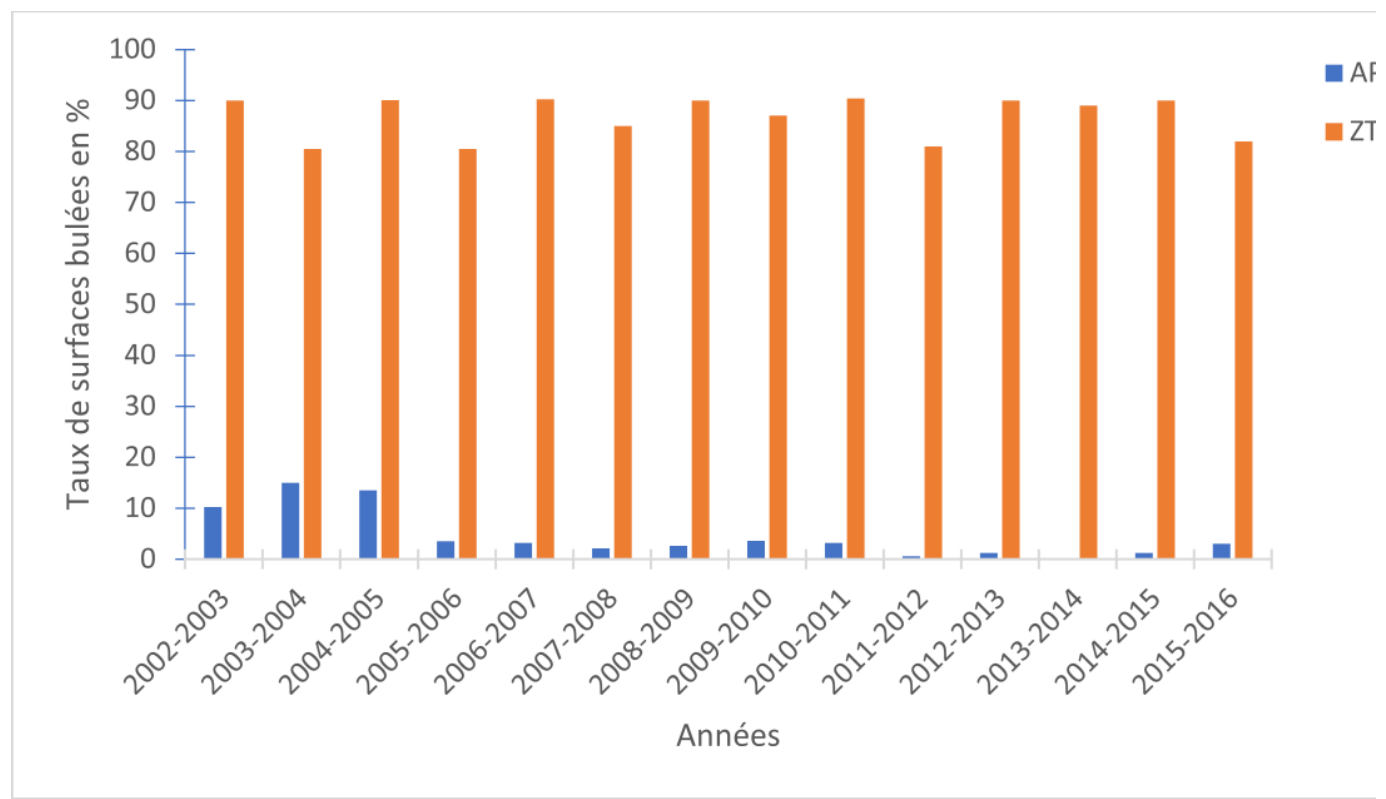

Figure 7: Evolution annuelle du taux de superficie brûlée dans le PNMD. AP : aire protégée, $Z T:$ zone tampon. 

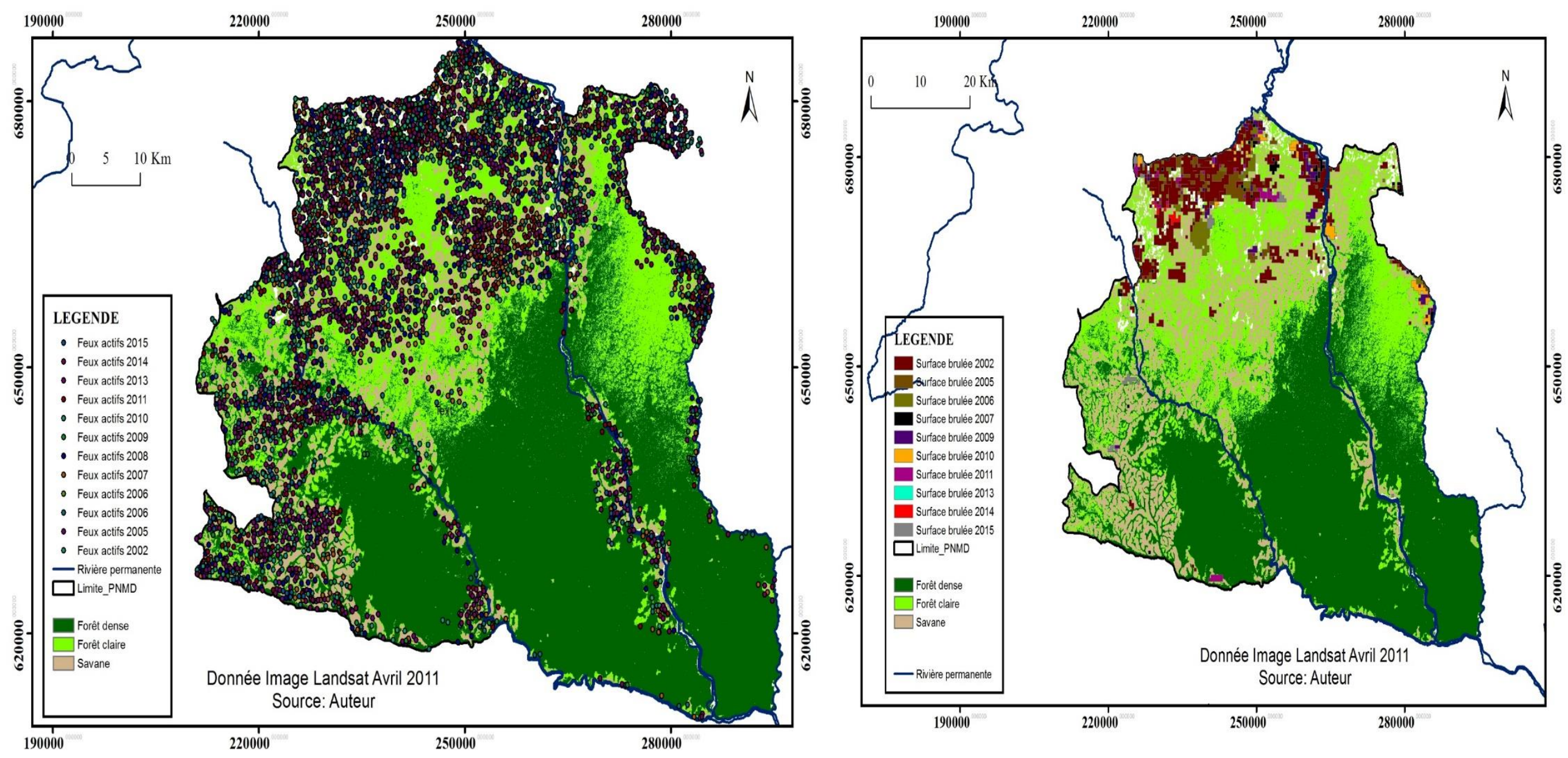

Figure 8 : Fréquence des feux actifs et superficie brûlée dans le PNMD entre 2002 à 2015. 

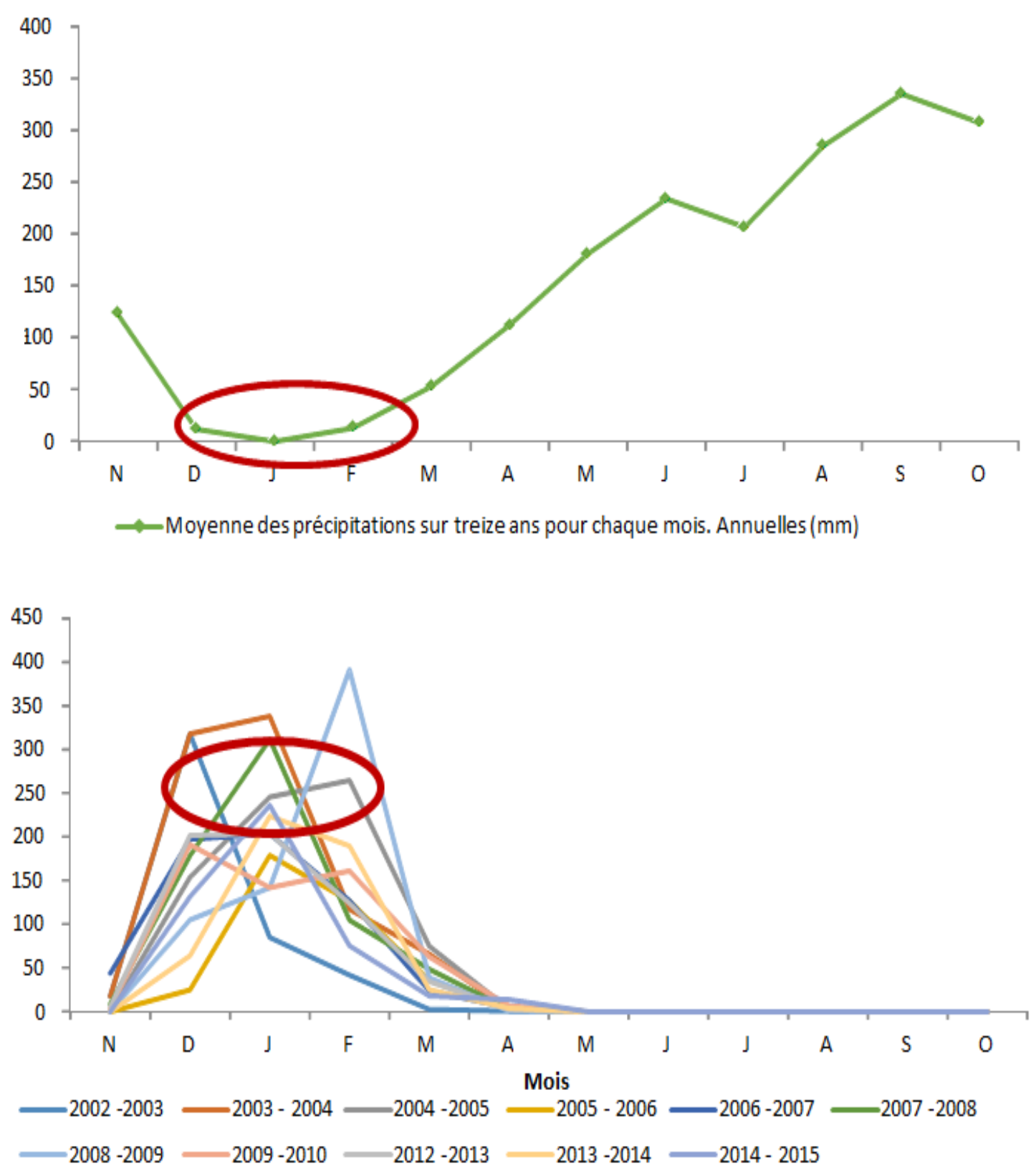

Figure 9: Courbe pluviométrique (en haut) et courbe des feux (en bas) dans le PNMD. 
C. D. GUEGUIM et al. / Int. J. Biol. Chem. Sci. 12(2): 728-748, 2018

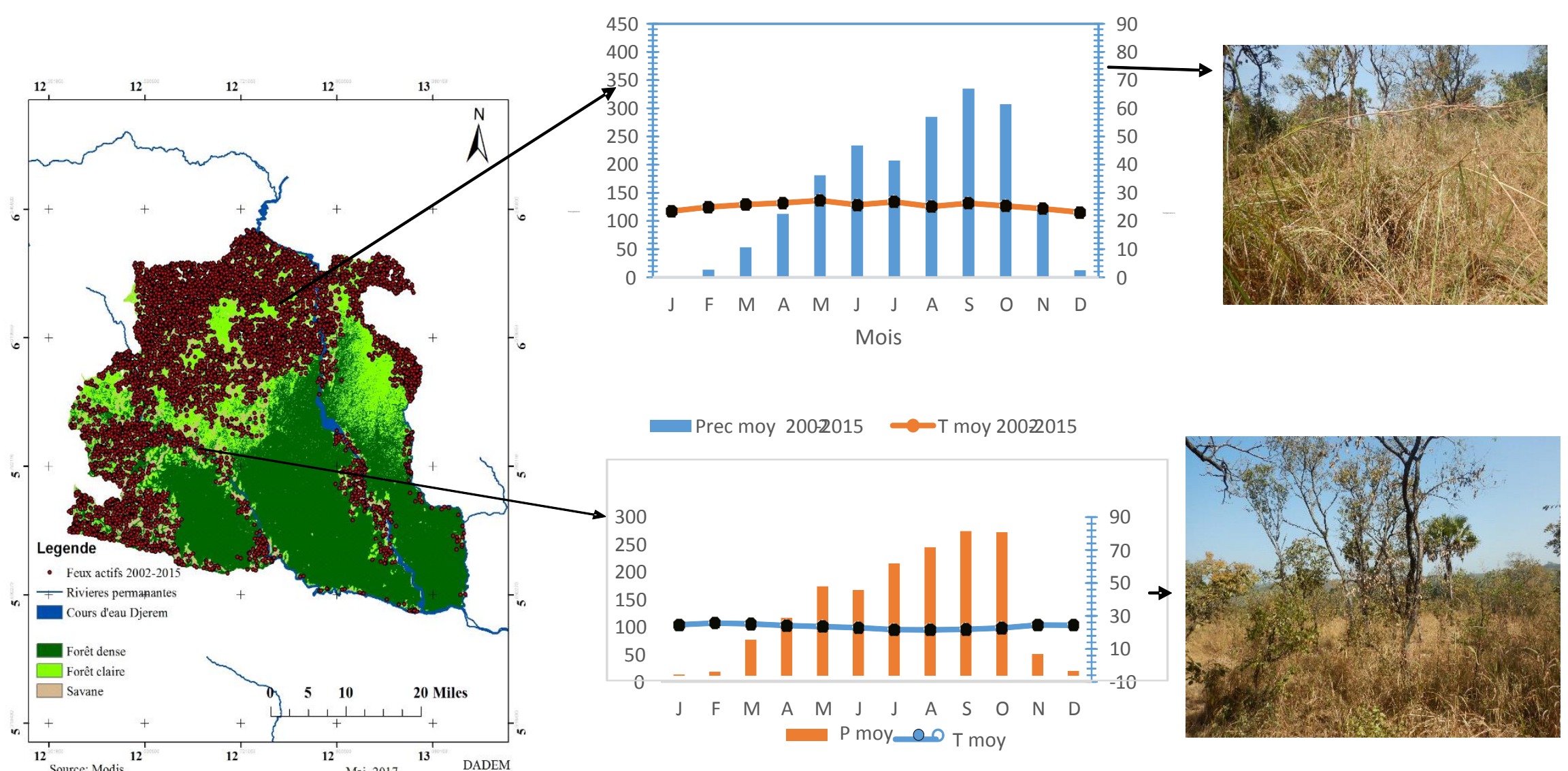

Figure 10: Feu de brousse, conditions climatiques et état du couvert végétal entre 2002 et 2015 dans le PNMD. 


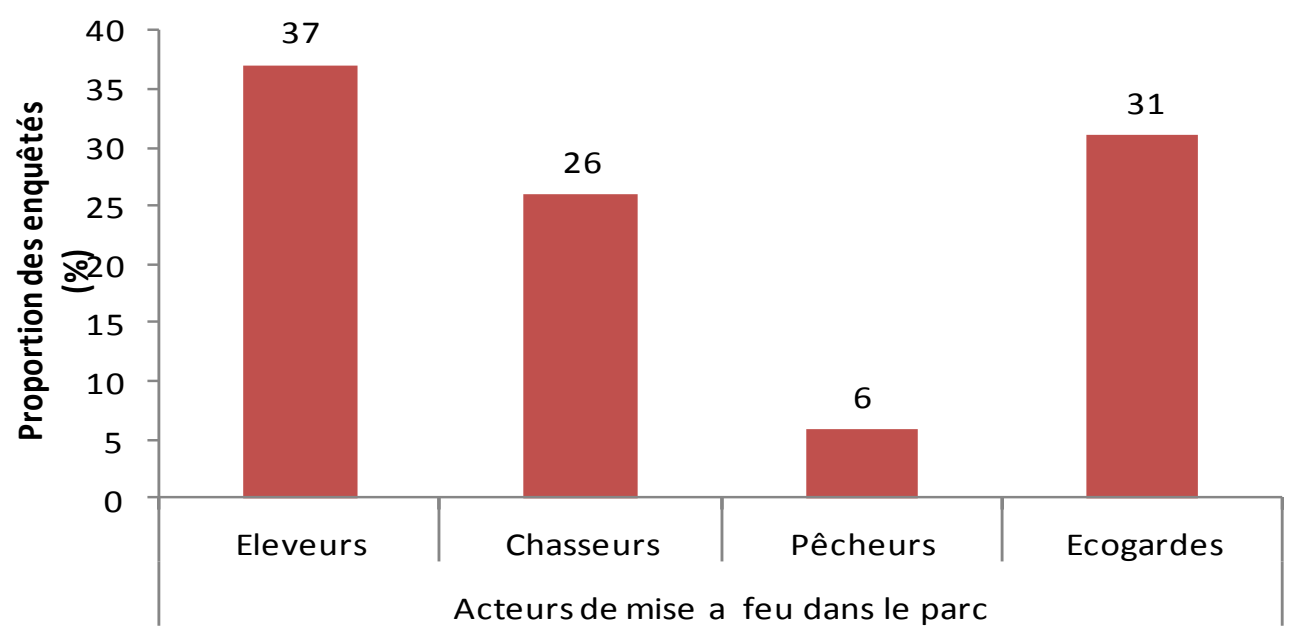

Figure 2: Proportions des acteurs de mis à feux de brousses dans le PNMD.

Tableau 2 Mois de mise à feu, type de feu, avantages pour les acteurs.

\begin{tabular}{|c|c|c|c|c|}
\hline $\begin{array}{l}\text { Mois de } \\
\text { mise à feu }\end{array}$ & $\begin{array}{l}\text { Type } \\
\text { de feu }\end{array}$ & Acteurs & Avantages pour les acteurs & $\begin{array}{l}\text { Inconvénients pour la } \\
\text { faune }\end{array}$ \\
\hline \multirow{2}{*}{$\begin{array}{l}\text { Novembre } \\
\text { - } \\
\text { Décembre }\end{array}$} & \multirow{2}{*}{$\begin{array}{l}\text { Feu } \\
\text { précoce }\end{array}$} & Éleveurs, & Obtenir les repousses & $\begin{array}{l}\text { Risque de contamination } \\
\text { de la faune }\end{array}$ \\
\hline & & Pécheurs & Nettoyer & $\begin{array}{l}\text { Extension non contrôlée } \\
\text { des flammes et destruction } \\
\text { de la faune }\end{array}$ \\
\hline \multirow{4}{*}{ Janvier } & \multirow{4}{*}{$\begin{array}{l}\text { Feu } \\
\text { tardif }\end{array}$} & Éleveurs, & Obtenir les repousses & \multirow{4}{*}{$\begin{array}{l}\text { Risque de contamination } \\
\text { de la faune } \\
\text { Extension non contrôlée } \\
\text { des flammes et destruction } \\
\text { de la faune }\end{array}$} \\
\hline & & Chasseurs ; & $\begin{array}{l}\text { - Rabattre le gibier; } \\
\text { - Ouvrir le champ de vision; } \\
\text {-Préparer les aires de chasse } \\
\text { future }\end{array}$ & \\
\hline & & & $\begin{array}{l}\text { - Renouveler la strate herbacée } \\
\text { pour la faune }\end{array}$ & \\
\hline & & Ecogardes & $\begin{array}{l}\text { Améliorer la visibilité pour le } \\
\text { travail des patrouilles anti- } \\
\text { braconnage }\end{array}$ & \\
\hline \multirow{4}{*}{$\begin{array}{l}\text { Févier- } \\
\text { mars et } \\
\text { avril }\end{array}$} & \multirow{4}{*}{$\begin{array}{l}\text { Feu } \\
\text { tardif }\end{array}$} & Éleveurs, & Obtenir les repousses & \multirow{4}{*}{$\begin{array}{l}\text { Risque de contamination } \\
\text { de la faune } \\
\text { Extension non contrôlée } \\
\text { des flammes et destruction } \\
\text { de la faune }\end{array}$} \\
\hline & & Chasseurs ; & Gibier & \\
\hline & & Ecogardes, & $\begin{array}{l}\text { Renouveler la strate herbacée } \\
\text { pour la faune, Améliorer la } \\
\text { visibilité pour le travail des } \\
\text { patrouilles anti-braconnage }\end{array}$ & \\
\hline & & Pêcheurs & Nettoyer & \\
\hline
\end{tabular}


Tableau 3 : Corrélations croisées entre l'indice de Thornthwaite (annuel) et la fréquence des feux/la surface brûlée annuelle dans le PNMD.

\begin{tabular}{llccccc}
\hline Périodes & Variables & \multicolumn{5}{c}{ Décalage (années avant le feu) } \\
\hline & & $\mathbf{0}$ & $\mathbf{1}$ & $\mathbf{2}$ & $\mathbf{3}$ & $\mathbf{4}$ \\
\hline \multirow{2}{*}{$\mathbf{2 0 0 2 - 2 0 0 4}$} & Fréquence & $-0.31^{*}$ & 0.19 & -0.09 & 0.18 & -0.12 \\
& Surface brûlée & $-0.36^{*}$ & 0.24 & -0.05 & 0.00 & 0.00 \\
\multirow{2}{*}{$\mathbf{2 0 0 5 - 2 0 1 5}$} & Fréquence & 0.03 & 0.01 & -0.16 & $0.33^{*}$ & -0.04 \\
& Surface brûlée & -0.04 & -0.08 & -0.05 & 0.05 & 0.02 \\
\hline
\end{tabular}

Les valeurs avec astérisques indiquent les corrélations significatives $(\mathrm{p}<0.05)$.

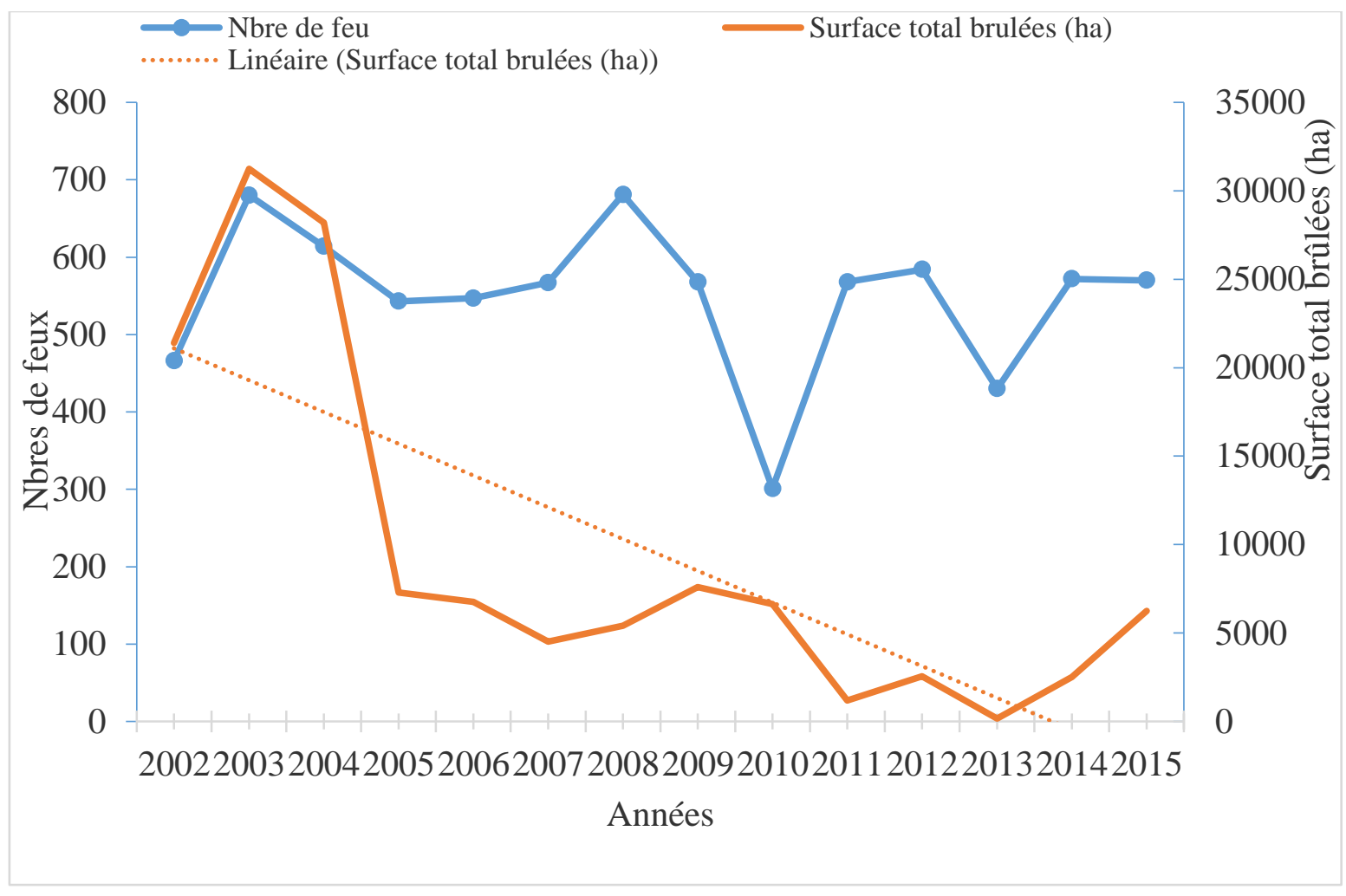

Figure 3: Fréquence des incendies et surfaces brûlées annuelles pour les périodes 2002 à 2015 dans le PNMD. 


\section{DISCUSSION}

Le suivi des données cumulées annuelles de feux actifs dans le PNMD a montré que la différence est significative d'une année à une autre. La tendance cyclique des feux extrêmes observée peut s'expliquer par le fait qu'après un feu catastrophique ayant décimé la biomasse une année donnée, l'écosystème mettrait 1 à 2 ans pour reconstituer le couvert végétal suffisant pour une seconde occurrence de feux intenses. Même si les graminées qui brûlent repoussent chaque année, ce qui permet le maintien des savanes par les feux, les mosaïques de paysages créées par les plages brûlées et non brûlées modifient le comportement du feu d'une saison à une autre. Les distributions patio temporelles des feux actifs et des superficies brûlées durant les saisons 20022003 jusqu'en 2015-2016 confirment que l'éclosion des feux a essentiellement lieu dans les savanes et les zones d'activités humaines contiguës à l'AP d'où se propagent ces feux vers d'autres formations végétales. Ainsi, les écosystèmes naturellement peu exposés aux feux (écotone, bosquets, forêts claires, forêt galeries et forêts denses sèches) peuvent, à l'échelle locale, être envahis par des feux à partir des savanes contiguës. Au niveau du parc, la répartition des spots de feux actifs à l'intérieur de l'AP semble longer les pistes d'intrusion et les cours d'eau dans les AP, ce qui traduit l'origine anthropique de la majorité des feux enregistrés à l'intérieur de cette AP. Les mêmes pistes sont empruntées par les agents forestiers lors de la pratique des feux d'aménagement et lors les patouilles antibraconnage, ce qui explique toujours cette relation entre les pistes forestières et les spots de feux actifs détectés. Ces remarques sont contraires aux résultats de Afelu et al. (2016) qui ont établi que 2 à 4 ans après un feu, l'écosystème reconstitue ses ressources végétales annulant pratiquement l'effet du feu antérieur.

L'analyse croisée de la localisation des feux actifs, de la distribution des superficies brûlées et de l'occupation au sol des différentes formations végétales (savanes et forêts) a permis de dégager la même information. Les feux de brousse concernent essentiellement les savanes, formations végétales ouvertes, à tapis graminéen ou herbacé continu et de ce fait plus exposées aux feux. Ces résultats se rapprochent de ceux obtenus par Opha (2009) qui a noté qu'au Botswana, les savanes sont les plus exposées aux feux et que les forêts sont peu concernées et ne subissent de véritables feux qu'à la haute dessiccation en fin de saison sèche ou en période de sécheresse exceptionnelle. Kana et Etouna (2006) avaient évalué, dans une étude au Cameroun sur la répartition des superficies brûlées, qu'à peine $7 \%$ concernaient les forêts et la forte proportion de $93 \%$ au niveau des savanes. Ainsi, la nature de la formation végétale et son occupation de sol sont des facteurs essentiels dans l'évaluation du niveau d'exposition et de vulnérabilité d'un écosystème au risque d'incendie (Palumbo et al., 2011).

D'autres auteurs (Koffi et al., 1995) ont constaté, après un suivi de la dynamique des feux sur quatre années en Afrique centrale, que l'écosystème le plus exposé est la savane tropicale dès que le contexte climatique est propice aux éclosions de feux. L'éclosion des feux a essentiellement lieu dans les savanes et les zones d'activités humaines contiguës aux AP (champs de culture sur brûlis, zones de chasse traditionnelle ou de carbonisation de bois, fermes) d'où se propagent ces feux vers les formations végétales relativement plus couvertes. En effet, au niveau du PNMD l'occupation humaine et la forte présence humaine au niveau de la zone tampon constituent des sources d'éclosion des feux et de leur propagation vers l'intérieur de l'aire protégée.

Au niveau du Sud, la proximité avec la zone de chasse protège l'intérieur du parc alors que les limites sont et ouest contigus aux localités riveraines sont des portes d'entrée des feux incontrôlés. Quant au nord du parc seule le fleuve Djerem au centre du parc constitue la barrière naturelle contre les feux extérieurs et la forte densité humaine dans cette zone essentiellement pêcheurs (pêche autorisé parc les gestionnaires du parc) 
explique l'intrusion dans le parc et l'éclosion des feux depuis l'intérieur de l'aire protégée. La distribution des feux actifs à l'intérieur des AP par rapport aux pistes d'intrusion dans les AP traduit l'origine anthropique de la plupart des feux remarquée par Hough (1993). Par conséquent, ce rapprochement entre les feux actifs et les pistes de traversé des AP tend non seulement à confirmer l'origine anthropique de ces feux mais aussi permet de cibler les zones à surveiller et à protéger en priorité (Bao et al., 2015 ; Bianchini et al., 2015). En somme, l'interdépendance entre le contexte sociologique et écologique et l'occurrence des feux est une évidence (Flannigan et Wotton, 2001) et la plupart des feux dans les savanes sont des feux volontaires d'origine humaine qui débordent et se propagent aux écosystèmes forestiers adjacents (Poilecot et Loua, 2009).

Les résultats et informations obtenus sur la dynamique des feux dans les AP et dans leurs zones périphériques respectives expriment la problématique de sécurisation de l'intérieur des AP par rapport à leurs ZT. En effet, une bonne sécurisation (délimitation consensuelle, matérialisée, aménagée et surveillée) permet d'y créer un tampon qui amortit l'avancée du stress extérieur (la propagation des feux éclos dans la ZT). La situation du PNMD est plus expressive. La gestion des terroirs et espaces agricoles ou d'habitation rurale de la zone périphérique vient en complément de sécurisation et d'aménagement de l'intérieur de l'AP. En somme, un faible niveau de sécurisation physique dans le contexte local de l'AP donne lieu aux intrusions et de délits dans l'AP et aux activités humaines sources d'éclosion de feu ou de sa propagation à partir des zones contiguës. Cette situation renforce les interfaces homme/AP, sources d'éclosion et de propagation des feux et de conflits d'accès aux ressources de l'AP. L'étude menée par Eriksen (2007) dans le parc national de South Luangwa en Zambie montre que la sécurisation de l'AP permet de protéger l'intérieur du parc (taux de SB $<8 \%$ ) des feux de sa ZT ou le taux de SB est compris entre 40 et $50 \%$. Ces résultats sont fondamentaux dans l'orientation stratégique de l'aménagement et la lutte contre les feux et surtout dans la planification à court et moyen termes de la gestion des feux de végétation. En effet, la nécessité du suivi et de l'analyse spatiotemporelle du phénomène au niveau sous régional ouest africain, national et local a été soulignée par Valea (2011) à travers une étude multi-échelles des feux au Burkina Faso. En effet, la connaissance de la dynamique spatiotemporelle des feux actifs et des superficies brûlées facilite la distribution stratégique des ressources de prévention et de lutte contre les feux et la planification objective de la surveillance des aires protégées (Bao et al., 2015).

\section{Conclusion}

L'état des lieux de la dynamique spatio-temporelle des feux dans le PNMD montre que la réglementation des feux n'a pas permis de réduire l'occurrence des feux tardifs et incontrôlés. Le risque demeure élevé et généralisé à une bonne partie du territoire. Le comportement du feu à l'intérieur de l'aire protégée est influencé par la gestion faite de ces feux au niveau de la zone tampon. Les superficies brûlées détectées par les satellites sont généralement sous estimées par rapport aux données enregistrées sur le terrain. En effet, les zones détectées comme non brûlées sont parsemées de plages brûlées et la superficie réellement brûlée est significativement plus importante que la superficie détectée. La sécurisation des limites d'une aire protégée peut faciliter la réduction de la propagation des feux des zones brûlées vers les zones protégées. La nécessité du suivi et de l'analyse spatio-temporelle des feux au niveau national, régional et local est souhaitable pour une organisation pratique de l'alerte précoce dans la prévention et la gestion des feux. Il découle de cette étude que la gestion des feux est un aspect fondamental et spécifique de l'aménagement du territoire et en particulier de la gestion des aires protégées.

\section{CONFLIT D'INTERETS}

Les auteurs déclarent qu'ils n'ont aucun conflit d'intérêts. 


\section{CONTRIBUTIONS DES AUTEURS}

Travail a été réalisé à $80 \%$ par CDG, $15 \%$ par NMT et $5 \%$ par CRF.

\section{REFERENCES}

Afelu B, Djongon KA, Kokou K. 2016. Dynamique spatiotemporelle des feux de végétation au Togo (Afrique de l'Ouest). European Scientific Journal, 12(18). DOI : 10.19044/esj. 2016.v12n18p14 URL http://dx.doi.org/10.19044/esj.2016.v12n $18 \mathrm{p} 14$.

Alvarado STE, Buisson H, Rabarison C, Rajeriarison C, Birkinshaw P, Lowry LPC. Morellato. 2014. Fire and the reproductive phenology of endangered Madagascar sclerophyllous tapia woodlands. South African Journal of Botany, 94: 79-87. DOI. http : //www.sciencedirect.com/ science/article/pii/s14609134218007885.

Alvarado ST. 2012. Evaluation du rôle des feux de brousse sur la composition, la structure, la phénologie, et la résistance de la végétation des bois de tapia (Uapaca bojeri) du massif d'Ibity, Nouvelle Aire Protégée, en vue de sa gestion durable. Thèse de Doctorat de l'Université d'Avignon et des Pays de Vaucluse, Avignon France, p 212.

Bianchini G, Caymes-Scutari P, MéndezGarabetti M. 2015. Evolutionary Statistical System: A parallel method for improving forest fire spread prediction. Journal of Computational Science, 6(15): 58-66. DOI : 10.1016/j.jocs.2014.12.001

Bao S, Ningchuan X, Zehui L, Heyuan Z, Changjoo K. 2015. Optimizing watchtower locations for forest fire monitoring using location models. Fire Safety Journal, 71: 100-109.

Collins Ross D, de Neuf ville R, Joao C, Tiago O, Abílio PP. 2013. Forest fire management to avoid unintended consequences: a case study of Portugal using system dynamics. Journal of Environmental Management, 130(30): 19. DOI: 10.1016/j.jenvman.2013.08. 033
Dolidon H. 2007. La multiplicité des échelles dans l'analyse d'un phénomène d'interface nature/société. L'exemple des feux de brousse en Afrique de l'ouest. Cybergeo, 8(363): www.cybergeo.eu/ pdf $/ 4805$

Devineau JL, Fournier A, Nignan S. 2010. Savanna fire regimes assessment with MODIS fire data: their relationship to land cover and plant species distribution in western Burkina Faso (West Africa). Journal of Arid Environments, 74: 10921101. doi:10.1016/j.jaridenv.2010.03.009

Eva HD, Grégoire J-M, Mayaux P, Cheva D. 2003. Suivi des Feux de Végétation dans les Aires Protégées d'Afrique SubSaharienne: Apport des Techniques Spatiales et des Données Satellitales pour le Suivi des Feux en Afrique SubSaharienne. Office for Officiai Publications of the European Communities: Luxembourg.

Eriksen C. 2007. Why do they burn the 'bush'? Fire, rural livelihoods, and conservation in Zambia. The Geographical Journal, 173(3): 242-256.

Flannigan MD, Wotton BM. 2001. Climate, Weather, and Area Burned. Forest Fires, Chapter, 10: 351-373.

Giglio L. MODIS Collection 5 Active Fire Product User's Guide Version 2.4. 2010. DOI:http://www.fao.org/fileadmin/templ ates/gfims/docs/MODIS_Fire_Users_Gu ide_2.4.pdf.

Grégoire JM, FournierA, Eva H, Sawadogo L. 2003. Caractérisation de la dynamique des feux et de l'évolution du couvert végétal dans le Parc du W : Burkina Faso, Niger et Bénin. Mission d'expertise pour l'étude des feux de brousse et leur utilisation dans le cadre d'une gestion raisonnée des aires protégées du complexe WAP, $64 \mathrm{p}$.

Guiguindibaye M, Belem OM, Boussim JI. 2013. Caractéristiques des feux dans un incendie en savane soudanienne au Tchad. Int. J. Biol. Chem. Sci., 7(3): 1147-1156. DOI: http://dx.doi.org/ 10.4314/ijbcs.v7i3.21 
Hough JL.1999. Why burn the bush? Social approaches to bush-fire management in West African national parks. Biological Conservation, 65(1): 23-28

Jacquin A. 2010. Dynamique de la végétation des savanes en lien avec l'usage des feux à Madagascar, Thèse de Doctorat de l'Université de Toulouse, p146.

Kana CE. 2009. Apport des données de télédétection dans la gestion des feux de végétation en territoire Camerounais, Thèse de Doctorat de l'Université de Toulouse, p312.

Kana EC, Etouna JE. 2006 Apport de trois méthodes de détection des surfaces brûlées par imagerie Landsat ETM+ : application au contact forêt- savane du Cameroun. Cybergeo, European Journal of Geography, http://cybergeo.revues. org/2711.

Kabore A. 2010. Brousse des uns, aire protégée des autres Histoire du peuplement, perceptions de la nature et politique des aires protégées dans le Gourma burkinabè: l'exemple de la Réserve partielle de faune de Pama ». Thèse de l'Institut de Hautes Etudes Internationales et du Développement. Genève, 386p.

Koffi B, Grégoire J-M, Mahé G, Lacaux J-P. 1995. Remote sensing of bush fire dynamics in Central Africa from 1984 to 1988: analysis in relation to regional vegetation and pluviometric patterns. Atmospheric Research, 39(1-3): 179200.
Ministère des Forêts et de la Faune (MINFOF). 2008. Plan d'aménagement du Parc National du Mbam et Djerem e de sa zone périphérique, Yaoundé, Cameroun : MINFOF.

N'dri AB. 2011. Interaction termite-feu et dynamique de la végétation en savane (LAMTO, Côte d'Ivoire). Thèse de doctorat, Université Paris 6, cotutelle avec l'Université d'Abobo-Adjamé, p. 175.

Opha PD. 2009. Linking fire and climate: interactions with land use, vegetation, and soil. Current Opinion in Environmental Sustainability, 1(2): 161169.

Palumbo I, Grégoire J-M, Simonetti D, Punga M. 2011. Spatio-temporal distribution of fire activity in protected areas of SubSaharan Africa derived from MODIS data. Procedia Environmental Sciences, 7: 26-31.

Poilecot P, Loua N-S. 2009. Les feux dans les savanes des monts Nimba, Guinée. Bois et Forêts des Tropiques, 301(3): 52-66,.

Valea F. 2011. Etudes des feux de brousse au Burkina Faso. Approche multi-échelles des feux actifs et des surfaces brûlées. Thèse de doctorat, Laboratoire GEOPHEN, Université de Caen Basse Normandie (France), 112 p.

Yameogo. 2005. Le feu, un outil d'ingénierie au Ranch de Gibier de Nazinga au Burkina Faso. Thèse de doctorat, Université d'Orléans, 268p. 